\title{
The clinical significance of upper gastrointestinal bleeding etiology score in determining variceal bleeding
}

\author{
Farhan Ali, Adnan Bashir Bhatti*, Siddique Akbar Satti, Tariq Mehmood Satti
}

Department of Internal Medicine, Capital Development Authority (CDA) Hospital, Islamabad, Pakistan

Email: *dr.adnanbashir@gmail.com

Received 23 December 2013; revised 25 December 2013; accepted 12 January 2014

Copyright (C 2014 Farhan Ali et al. This is an open access article distributed under the Creative Commons Attribution License, which permits unrestricted use, distribution, and reproduction in any medium, provided the original work is properly cited. In accordance of the Creative Commons Attribution License all Copyrights (C) 2014 are reserved for SCIRP and the owner of the intellectual property Farhan Ali et al. All Copyright (C) 2014 are guarded by law and by SCIRP as a guardian.

\section{ABSTRACT}

BACKGROUND: Upper gastrointestinal bleeding (UGIB) is a common, and potentially life threatening condition, which can be divided into variceal and non-variceal sources of bleeding. OBJECTIVE: To examine the validity of the upper gastrointestinal bleeding etiology score compared to the current gold standard, the emergency Esophagogastroduodenoscopy (EGD), for determining the etiology of UGIB. METHODOLOGY: 101 patients presenting with upper gastrointestinal bleeding presented in the emergency department of Capital Hospital, Islamabad between February 2010 and March 2012 were included in this cross-sectional study. The upper gastrointestinal bleeding score was computed for each case by accounting for the clinical parameters of previous diagnosis of cirrhosis or signs of chronic liver disease $\times 3.1$, presence of red vomitus $\times 1.5$, and red N/G aspirate $\times 1.2$. Each parameter was given a score of 1 if present, and 0 if absent, with a total score $\geq 3.1$ favoring variceal bleed, and a score of $<3.1$ indicating non-variceal bleeding as a cause of UGIB. Esophagogastroduodenoscopy was performed within 72 hours of presentation. RESULTS: The mean \pm SD age of the patients was $50.2 \pm 14.1$ years ranging from 18 to 80 years. Out of 101 patients, $56 \%$ were males while the remaining $44 \%$ were females. The sensitivity of the UGIB score was $78.2 \%$ and the specificity was $84.3 \%$. The positive predictive value (PPV) was $91.5 \%$ and negative predictive value (NPV) was $64.2 \%$. The overall diagnostic accuracy of UGIB score in determining variceal bleeding was found to be $80.2 \%$. CONCLUSION: Variceal bleeding is a common cause of UGIB in Pakistan. UGIB etiology score is a highly sensitive and specific clinical tool in determining the

*Corresponding author. etiology of UGIB as either variceal or non-variceal bleeding.

\section{KEYWORDS}

Upper Gastrointestinal Bleeding; Upper

Gastrointestinal Bleeding Etiology Score; Variceal

Bleeding; Non-Variceal Bleeding; Endoscopy

\section{INTRODUCTION}

Upper gastrointestinal bleeding (UGIB) is defined as bleeding from a source proximal to the ligament of Treitz, differentiating it from lower gastrointestinal bleeding, which involves the colon, the middle gastrointestinal bleeding and the small intestine distal to the ligament of Treitz [1]. The incidence of UGIB is reported to be approximately 100/100,000 per annum worldwide, and it is approximately 4 times as common as lower GI tract bleeding [2]. It is a major cause of morbidity and mortality, with overall mortality rates ranging from $6 \%-10 \%$ [2].

UGIB can be divided into variceal and non-variceal sources of bleeding. Variceal bleeding has been very extensively studied in patients suffering from liver cirrhosis but even so, about 30\% - 40\% cirrhotic patients presenting with bleeding GI tracts may in fact have nonvariceal UGIB etiology, a condition frequently caused by peptic ulcers [3].

Cirrhosis of the liver is a major cause of portal hypertension, which may in turn result in the development of esophageal varices (EV) [4]. In Pakistan, the incidence of hepatitis, as well as the subsequent development of cirrhosis, is relatively high, with management of portal hypertension and its associated symptoms posing a serious problem for physicians [5].

In patients with UGIB, endoscopy is traditionally used 
to diagnose most causes of UGIB, and has, especially when performed within the first 24 hours of the hospital stay, been associated with a reduction in blood transfusion requirements, and in the length of intensive care unit and total hospital stay [6].

The aim of this study was to validate the UGIB etiology score for determining the cause of UGIB as variceal vs. non-variceal, by taking into account a number of clinical parameters and comparing it to the gold standard, esophagogastroduodenoscopy (EGD).

\section{METHODOLOGY}

\subsection{Overview}

This study was conducted in the emergency department of medicine at Capital Hospital, Islamabad, Pakistan, which is a tertiary care hospital, from January 1, 2011 to December 31, 2012, after receiving permission from the concerned authorities of Capital Hospital Islamabad (the Head of the Department of Medicine, Hospital Ethical Committee).

\subsection{Sample Size and Sampling Technique}

By non-probability convenient sampling, 101 patients of confirmed upper gastrointestinal bleeding (UGIB) aged $>18$ years were included in the study. Patients were diagnosed with UGIB, based on the presence of hematemesis, melena or hematochezia, and/or a positive N/G tube aspiration for coffee-ground, black or bloody contents.

\subsection{Data Collection}

Verbal consent was taken from all patients after explaining the nature and purpose of the study. All patients were handled by the same doctor to minimize bias. Patients with upper gastrointestinal bleeding were identified, and detailed history was taken for diagnosis and for fulfillment of the required selection criteria.

\subsection{Tools}

Using structured proforma, information was collected. Information regarding baseline characteristics of the patients was collected first; their age and sex, were noted. By vigorous and strict criteria, only patients presenting with confirmed upper gastrointestinal bleeding were selected. Patient detailed history was taken; general physical examinations and detailed systemic examinations were performed.

The upper gastrointestinal bleeding (UGIB) etiology score was computed for each case and patients were classified as suffering from variceal bleeding if the score was $\geq 3.1$ and non-variceal bleeding if the score was $<3.1$. To calculate the score, a previous diagnosis of cirrhosis or the presence of signs of chronic liver disease (palmer erythema, spider nevi, bruises, gynecomastia, ascites, splenomegaly, ankle edema, testicular atrophy, confusion and drowsiness) were scored as 1 if present and 0 if absent; red vomitus was scored 1 if present and 0 if absent; and red Nasogastric (N/G) aspirate was scored 1 if present and 0 if absent. Upper GI endoscopy was performed by the consultant within 72 hours after onset of UGIB symptoms for all patients, to look for variceal or non-variceal bleeding as the cause of UGIB.

\subsection{Data Analysis Procedure}

The statistical package for social sciences (SPSS, version 16.0, Chicago, IL) was used to enter and analyze the data. Sensitivity and specificity values of UGIB etiology score were calculated.

\subsection{Exclusion Criteria}

Patients presenting after 72 hours of upper gastrointestinal bleeding as well as patients unfit or uncooperative for upper gastrointestinal endoscopy, were excluded from the study.

\section{RESULTS}

In this study, a total of 101 patients with upper gastrointestinal bleeding (UGIB) were included. 56\% of these were male, and $44 \%$ female. The mean \pm standard deviation (SD) age of the study patients was $50.2 \pm 14.1$ years ranging from 18 to 80 years. Approximately $75 \%$ of the study population was $>41$ years of age, while $3 \%$ and $7 \%$ were in their $2^{\text {nd }}$ and $3^{\text {rd }}$ decades of life, respectively.

The main clinical feature observed was presence of red vomitus as listed in Table 1. Out of the total 101 cases, most patients were diagnosed as having grade 3 esophageal varices, with all endoscopy findings listed in Table 2.

We here found that according to the UGIB etiology score in determining variceal bleeding using endoscopy as gold standard, the values of true positive was 54 (78.3\%) as listed in Table 3. The overall diagnostic accuracy of the UGIB score in determining variceal bleeding was found to be $80.2 \%$ as listed in Table 4 , whereas the UGIB score in diagnosing variceal bleeding is listed in Table 5.

Table 1. Clinical features of UGIB patients $(n=101)$.

\begin{tabular}{ccc}
\hline & Number & Percentage \\
\hline $\begin{array}{c}\text { Previous diagnosis of cirrhosis or } \\
\text { signs of CLD }\end{array}$ & 59 & $58.4 \%$ \\
$\begin{array}{c}\text { Presence of red vomitus } \\
\text { Presence of red N/G aspirate }\end{array}$ & 73 & $72.3 \%$ \\
\hline
\end{tabular}


Table 2. Endoscopic findings in UGIB patients $(n=101)$.

\begin{tabular}{ccc}
\hline & Number & Percentage \\
\hline Grade 1 esophageal varices & 18 & $17.8 \%$ \\
Grade 2 esophageal varices & 15 & $14.8 \%$ \\
Grade 3 esophageal varices & 36 & $35.6 \%$ \\
Esophagitis & 6 & $5.9 \%$ \\
Gastritis & 6 & $5.9 \%$ \\
Ulcer/gastric erosion & 4 & $3.9 \%$ \\
Duodenitis & 3 & $2.9 \%$ \\
Gastropathy & 2 & $1.9 \%$ \\
\hline
\end{tabular}

Table 3. Distribution of true positive, false positive, false negative and true negative values of the upper gastrointestinal bleeding etiology score according to endoscopic findings in the study $(\mathrm{n}=101)$.

\begin{tabular}{rcc}
\hline & Number & Percentage \\
\hline True positive & 54 & $78.3 \%$ \\
False positive & 5 & $15.6 \%$ \\
False negative & 15 & $21.7 \%$ \\
True negative & 27 & $84.4 \%$ \\
\hline
\end{tabular}

Table 4. Values of sensitivity, specificity, positive predictive value and negative predictive value of upper gastrointestinal bleeding etiology score in diagnosing variceal bleeding using endoscopy as gold standard $(\mathrm{n}=101)$.

\begin{tabular}{cc}
\hline & Value (\%) \\
\hline Sensitivity & $78.2 \%$ \\
Specificity & $84.3 \%$ \\
Positive predictive value (PPV) & $91.5 \%$ \\
Negative predictive value (NPV) & $64.2 \%$ \\
Overall diagnostic accuracy & $80.2 \%$ \\
\hline
\end{tabular}

Table 5. Values of $2 \times 2$ table of UGIB score in diagnosing variceal bleeding using endoscopy as gold standard $(n=101)$.

\begin{tabular}{cccc}
\hline \multirow{2}{*}{$\begin{array}{c}\text { UGIB } \\
\text { score }\end{array}$} & \multicolumn{2}{c}{ Endoscopy findings } & Total \\
\cline { 2 - 3 } & Variceal bleeding & Non-variceal bleeding & \\
\hline \multirow{2}{*3.1}{} & (a) & (b) & 59 \\
& 54 & 5 & \\
$<3.1$ & (c) & (d) & 42 \\
& 15 & 27 & \\
& (a + c) & (b + d) & \\
& 69 & 32 &
\end{tabular}

\section{DISCUSSION}

Upper gastrointestinal bleeding is a very common condition worldwide [1]. The current choice of investigation for diagnosing UGIB is endoscopy, which plays a crucial role in the screening and therapy of UGIB, and has been shown to not only reduce the overall mortality, rebleeding risk and transfusion requirements, but also the costs associated with the condition [7-10].

Since esophagogastroduodenoscopy is not always readily available, and since there is a lack of trained workforce, it is necessary to develop approaches that can differentiate between variceal and non-variceal bleeding other than EGD, in order for its management to be done timely and with appropriate treatment modality. Our current study was done to see the diagnostic validation of one such tool; the upper gastrointestinal bleeding etiology score.

Pongprasobchai et al. [11] showed in their study that the average age for UGIB was 53 years for variceal bleeding, and 61 years for non-variceal bleeding. Likewise, Tammaro L. et al. [12] also revealed an older age of their patients; the mean age of their patients was 65 years. Similarly, our study showed that the majority of UGIB cases presented in the later stages of life.

It has been reported previously that UGIB patients are predominantly male (ranging between $60 \%$ - 80\%) [11, $12]$, and comparably, in our study, we found that $56 \%$ of all patients were male.

We moreover found that the diagnostic validity of the UGIB score was highly valid in screening patients with variceal bleeding. The sensitivity, specificity, positive predictive value and negative predictive value of the UGIB etiology score in predicting variceal bleeding was $78.2 \%, 84.3 \%, 91.5 \%$ and $64.2 \%$ respectively. The overall diagnostic accuracy of UGIB score in the current study was $80.2 \%$. Pongprasobchai and colleagues reported a somewhat similar validity of the UGIB etiology score in their study: while keeping the cut off value of $\geq 3.1$ as positive; they reported sensitivity, specificity, positive predictive value and negative predictive values of $85 \%, 82 \%, 50 \%$ and $96 \%$, respectively [11]. The overall diagnostic accuracy was comparable with our study, at $81 \%$ [11].

The strong validity of the UGIB etiology score indicates a potential for it, as a pre-endoscopy confirmatory tool for variceal bleeding. Though endoscopy is the gold standard for diagnosing UGIB, it is however associated with some limitations, including: aspiration; adverse reactions to conscious sedation; perforation; and increasing bleeding while attempting therapeutic intervention, and the risk of complications increase with the presence of a serious medical condition, such as ventricular tachycardia, near respiratory arrest or hypotension [13]. 
There have been variable reports on the incidence of variceal bleeding from $6 \%$ to $14 \%$, and one study even reported an incidence of $45.7 \%$ [5]. Comparably, in our study the incidence of variceal bleeding was very high, and we found that almost $68 \%$ patients were diagnosed as having variceal bleeding by endoscopy.

In the current study, presence of cirrhosis or signs of CLD were positive in $58.4 \%$ of patients. This finding is comparable to other reports, which have revealed that between $50 \%$ - $60 \%$ of cirrhotic patients with UGIB have a greater risk of variceal bleeding [14-16].

According to the above-mentioned studies, and as confirmed by our findings, the UGIB etiology score has a high validity in predicting variceal bleeding in patients with upper gastrointestinal bleeding. As the EGD facility is not easily accessible in all health care services, the preendoscopy clinical scores can be used to determine the origin of the UGIB. Thus, the patients with variceal bleeding can be provided with more specific initial treatment, and avoid the unnecessary delays that are associated with the specific treatments, whereas the stable patients with non-variceal bleeding can be managed as outpatients, reducing the hospital burden and costs.

\section{CONCLUSION}

We conclude that, in accordance with our study findings, the UGIB etiology score is a highly sensitive and specific tool that can and should be used clinically to determine the etiology of UGIB as variceal or non-variceal bleeding. In our study, the overall diagnostic accuracy of the UGIB score is in conformity with previous reports on this topic. We suggest that in the facilities where EGD services are not available, the UGIB etiology score can safely be introduced, in order to help decide the initial empirical treatment offered to the patients presenting with UGIB, and to avoid unnecessary delays and reduce the costs of hospitalization and medications, by managing the stable patients with non-variceal bleeding as outpatients. This is one of the very few studies of its origin done in Pakistan. Very limited evidence is currently available on the diagnostic strength of the UGIB etiological score, and we hope that this study will provide insight and further information regarding variceal and nonvariceal upper gastrointestinal bleeding to local, as well as international, investigators and health providers working in gastroenterology.

\section{REFERENCES}

[1] Cappell, M.S. and Friedel, D. (2008) Initial management of acute upper gastrointestinal bleeding: From initial evaluation up to gastrointestinal endoscopy. Medical Clinics of North America, 92, 491-509. http://dx.doi.org/10.1016/j.mcna.2008.01.005
[2] Geibel, J. (2011) Upper gastrointestinal bleeding. http://emedicine.medscape.com/article/187857-overview

[3] González-González, J.A., et al. (2011) Non-variceal upper gastrointestinal bleeding in patients with liver cirrhosis. Clinical features, outcomes and predictors of in-hospital mortality. A prospective study. 10, 287-295.

[4] Ismail FW, Shah HA, et al. Noninvasive predictors of large varices in patients hospitalized with gastroesophageal variceal haemorrhage. 2008;2(1):124-8.

[5] Hamid, R.S., Haq, T.U., et al. (2011) Transjugular intrahepatic portosystemic shunt (TIPS); Review of initial experience at Aga Khan University Hospital. Journal of Pakistan Medical Association, 61, 336-339.

[6] Adler, D.G., Leighton, J.A., Davila, R.E., et al. (2004) ASGE guideline: The role of endoscopy in acute nonvariceal upper-GI hemorrhage. Gastrointestinal Endoscopy, 60, 497-504.

[7] Church, N.I., Dallal, H.J., Masson, J., et al. (2006) Validity of the Rockall scoring system after endoscopic therapy for bleeding peptic ulcer: A prospective cohort study. Gastrointestinal Endoscopy, 63, 606-612. http://dx.doi.org/10.1016/j.gie.2005.06.042

[8] Zappa, M., Visioli, C.B., Ciatto, S., et al. (2007) Gastric cancer after positive screening faecal occult blood testing and negative assessment. Digestive and Liver Disease, 39, 321-326. http://dx.doi.org/10.1016/j.dld.2006.11.010

[9] Manner, H., May, A., Faerber, M., et al. (2006) Safety and efficacy of a new high power argon plasma coagulation system (hp-APC) in lesions of the upper gastrointestinal tract. Digestive and Liver Disease, 38, 471-478. http://dx.doi.org/10.1016/j.dld.2006.03.022

[10] Thomopoulos, K., Theocharis, G., Mimidis, K., et al. (2006) Improved survival of patients presenting with acute variceal bleeding. Prognostic indicators of shortand long-term mortality. Digestive and Liver Disease, 38, 899-904. http://dx.doi.org/10.1016/j.dld.2006.08.002

[11] Pongprasobchai, S., Nimitvilai, S., Chasawat, J., et al. (2009) Upper gastrointestinal bleeding etiology score for predicting variceal and non-variceal bleeding. World Journal of Gastroenterology, 15, 1099-1104. http://dx.doi.org/10.3748/wjg.15.1099

[12] Tammaro, L., Di Paolo, M.C., Zullo, A., et al. (2008) Endoscopic findings in patients with upper gastrointestinal bleeding clinical classified into three risk groups prior to endoscopy. World Journal of Gastroenterology, 14, 5046-5050.

[13] Cappell, M.S. and Iacovone Jr., F.M. (1999) Safety and efficacy of esophagogastroduodenoscopy after myocardial infarction. The American Journal of Medicine, 106, 2935. http://dx.doi.org/10.1016/S0002-9343(98)00363-5

[14] Del Olmo, J.A., Pena, A., Serra, M.A., et al. (2000) Predictors of morbidity and mortality after the first episode of upper gastrointestinal bleeding in liver cirrhosis. Journal of Hepatology, 32, 19-24. http://dx.doi.org/10.1016/S0168-8278(01)68827-5

[15] Afessa, B. and Kubilis, P.S. (2000) Upper gastrointestinal bleeding in patients with hepatic cirrhosis: Clinical course 
and mortality prediction. The American Journal of Gastroenterology, 95, 484-489.

http://dx.doi.org/10.1111/j.1572-0241.2000.01772.x

[16] Lecleire, S., Di Fiore, F., Merle, V., et al. (2005) Acute upper gastrointestinal bleeding in patients with liver cirr- hosis and in noncirrhotic patients: Epidemiology and predictive factors of mortality in a prospective multicenter population-based study. Journal of Clinical Gastroenterology, 39, 321-327.

http://dx.doi.org/10.1097/01.mcg.0000155133.50562.c9 